\title{
Study of Current Distribution over a Power Cable Presenting Non-Uniform Geometry using the Partial Differential Equations Approach
}

\author{
G. Bousaleh author ${ }^{2}$, F. Hassoun ${ }^{1}$ and M. Akoum ${ }^{2}$. \\ ${ }^{1}$ France Telecom R\&D - France \\ 2 Lebanese University- IUT Saida - Lebanon \\ e-mail: gbousaleh@ hotmail.fr
}

\begin{abstract}
This paper presents a theoretical study of the currents and voltages characteristics transmission over an energy cable (Power Line Transmission PLT). The geometry of line is non-uniform. The proposed approach is based on the Distributed Network model, where the lumped parameters vary as the line's geometry.

Most of the numerical methods applied use a frequency approach. However, other techniques are available in the time domain that are more applicable when the perturbation is transitory or an impulse.

The partial differential equations (PDE) of telegraphers with variable coefficients are used in order to model the transmission over non-uniform lines.

This paper presents the adaptation and application of the FDTD method to solve the problem of transmission of an electric wave over a transmission line whose electromagnetic topology is nonuniform.

As an example, the final part of the paper proposes the modeling of the transmission of a transitory wave over an electric power cable. We present an application in the case of the propagation of lightning on an energy cable composed of three twisted wires. The validation of the proposed method will be set by comparing the obtained results with those obtained from a simulation in the frequency domain followed by an IFFT.
\end{abstract}

\section{Keywords}

Power Line Transmission (PLT), Finite Difference Time Domain, PDE, Electric wave propagation, Lightning.

\section{Introduction}

The recent trends in electronics relying on high frequencies show problems of electromagnetic interferences. In fact, a transmission system has physical links that allow the transmission of information between the different subsystems: It is crucial to evaluate the capability of these links in supporting such high frequencies without distorting the electromagnetic environment.

For example, the Power Line Transmission (PLT) technology that uses the existent electric network uses frequencies less that $30 \mathrm{MHz}$. Thus, it is convenient to offer new services relying on these new technologies and respecting the EMC regulations.

For such systems, it is convenient not only to characterize the copper medium at high frequency but also to study the problem of electromagnetic compatibility: the signal transmission must be carried without distortions or perturbations.

The transient's phenomena are frequent on the transmission lines, the first studies of these phenomena go back to studying the issue of rectangular pulses used in telegraphy. Moreover, the transient's noises may be unacceptable defects. In particular, a rapid change of load on an electrical distribution line or telephone line, produce a transient phenomenon which propagates along the line, even a lightning striking a transmission line induces a strong electromagnetic wave disturbance that will spread in the network.

The study of electromagnetic compatibility phenomena can be carried out using the transmission line theory. The method efficiency is strongly tied to the linear parameters (P.U.L. parameters) of the different sections which constitute the elementary structures of the equivalent model.

In this paper we propose an approach to simulate the propagation of a disturbance on an electrical transmission line. We calculate in the time domain the current values $\mathrm{i}(\mathrm{x}, \mathrm{t})$ and voltage $\mathrm{v}(\mathrm{x}, \mathrm{t})$ at each position on the line. The simulation code is performed on the MATLAB software.

In the first part we propose a model of the transmission nonuniform line. We detail the setting of the chosen model equation. The second part is a presentation of the implementation of the FDTD method for solving these equations. The third part is a graphic illustration of the results with a study of the influence of electrical parameters of the line on the propagation phenomenon.

\section{Model of a transmission line (TL)}

A transmission line is a set of conductors that allows the transmission of information from a generator to a receiver.

In $\mathrm{HF}$, the propagation phenomenon must be taken into account. In addition the energy does not flow exclusively in the wires but also spreads into the surrounding environment. Each wire behaves as an antenna. Quantifying the radiated electromagnetic energy requires the calculation of voltage and current for each position on the line and for each moment. Through the study of 
transmission of data over a network line power supply (PLT) this paper proposes a model of a nonuniform line based on the modified theory of lines. The electrical parameters are not constant and varies along the line depending on its geometry

A transmission line can be fragmented into a series of elementary section. These sections have a length $\mathrm{dx}$ small compared to the wavelength.

The representation of an elementary section is:

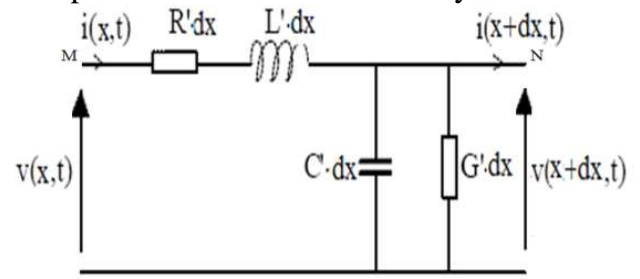

Fig. 1. Elementary section of a transmission line

We assume that per unit length parameters are independent of frequency but may depend on the position $\mathrm{x}$ on the line Ohm's law allows to write:

$R^{\prime} d x . i(x, t)+L^{\prime} d x \frac{\partial i(x, t)}{\partial t}=-\partial v(x, t)$

$C^{\prime} d x \cdot \frac{\partial v(x, t)}{\partial t}+G^{\prime} d x \cdot v(x, t)=-\partial i(x, t)$

The equations of a transmission line in the time domain are known as the telegrapher equations:

$$
\begin{cases}\frac{\partial v(x, t)}{\partial x}+R^{\prime} i(x, t)+L^{\prime} \frac{\partial i(x, t)}{\partial t}=0 & (\mathrm{I}-\mathrm{A}) \\ \frac{\partial i(x, t)}{\partial x}+G^{\prime} v(x, t)+C^{\prime} \frac{\partial v(x, t)}{\partial t}=0 & (\mathrm{II}-\mathrm{A})\end{cases}
$$

3-Finite difference method applied to solving the equations of line.

There are several analytical or numerical methods to calculate the distribution of current and voltage along a transmission line, each being better adapted to the type of electrical disturbance.

Most modeling methods use a frequency approach. However in the case of a disturbance type impulse or transient, we chose a numerical technique developed in the time domain FDTD (Finite Difference Time Domain) or finite difference method in time domain. We quote such types of disturbances a lightning on a power line used for transmission (PLT) and the impulsive noise on a telephone line. In this section we present the FDTD method, and then we present the application of this method to resolve equations of a transmission nonuniform line.

\section{Presentation of the FDTD method}

Since the late 80 s, there is an exceptional development of the numerical computation methods "PDE" (Partial Differential Equation). These methods solve partial differential equations in time domain or frequency. The reasons for using these methods are multiple

- Robustness of the calculations for a wide variety of systems

- Possibility of avoiding matrix inversions (otherwise, the matrices are only partially fulfilled)

- Systematic approach to all types of structures,

- Very high growth of IT resources that enable such methods to evolve very quickly.

- Easy visualization of many physical phenomena.

The FDTD method, proposed by Kane Yee in 1966, is interesting in many application areas. This interest is in the variety of structures can be modeled and the level of frequency ranges explored.

The FDTD has been applied to many fields in electromagnetism:

- study the response of the human body to electromagnetic radiation

- solving Maxwell's equations.

- Simulation of antennas (antennae micro ribbon, antenna array ...)

- Study of reverberant rooms .....

The method involves replacing the partial derivatives of a PDE by differences between values of the desired function in a finite number of discrete points (mesh nodes).

Let $\mathrm{F}$ be a function defined, continuous and differentiable in the neighborhood of $\mathrm{x} 0$, the derivative of the function $\mathrm{F}$ at $\mathrm{x} 0$ is obtained from the development in Taylor series of $\mathrm{F}$ at points $\mathrm{x} 0+\Delta \mathrm{x} / 2$ and $\mathrm{x} 0-\Delta \mathrm{x} / 2$

$$
F^{\prime}\left(x_{0}\right)=\frac{F\left(x_{0}+\frac{\Delta x}{2}\right)-F\left(x_{0}-\frac{\Delta x}{2}\right)}{\Delta x}+\theta\left(\Delta x^{3}\right)
$$

\section{A. Application of the FDTD method to equations of a uniform transmission line}

The application of the finite difference method to these equations begins with the discretization of the transmission line and the approximation of partial differential equations governing the system by centered finite differences, And then the resolution of the system of equations, taking into account the boundary conditions and initial conditions, gives $v(x, t)$ and $i(x, t)$.

The spatial discretization method for defining nodes voltages and currents is illustrated by the following figure. The wire is subdivided in turn into current and voltage nodes. The two ends of the line are defined as voltage nodes

Similarly, time can be discretized into intervals $\Delta \mathrm{t}$. 
The voltage $\mathrm{V}$ is calculated at times $\mathrm{n} \Delta \mathrm{t}$ and the current $\mathrm{I}$ at times $(n+1 / 2) \Delta t$.

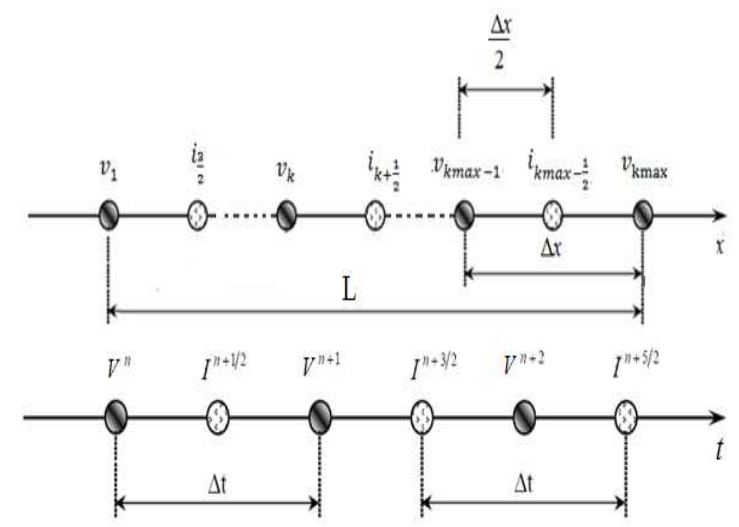

Fig. 2. Spatial and temporal discretization of TL

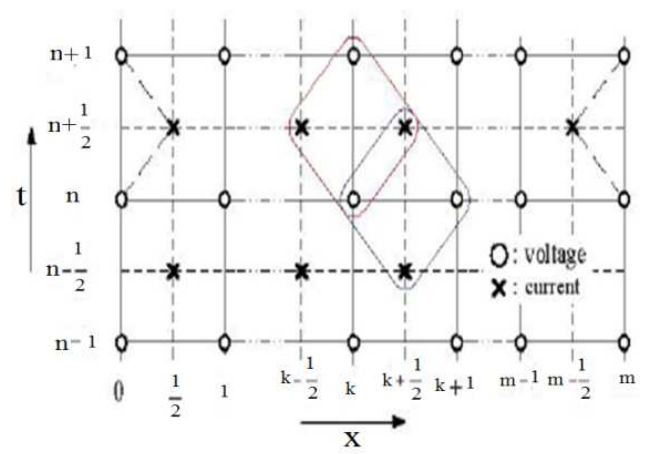

Fig. 3. Schema of TL after discretization

If we apply the principle of centered derivative the equations (I) (II) become:

$\frac{v_{k+1}^{n}-v_{k}^{n}}{\Delta x}+R \frac{i^{n+\frac{1}{2}}+i_{k+\frac{1}{2}}^{n-\frac{1}{2}}}{2}+L \frac{i^{n+\frac{1}{2}}-i^{k+\frac{1}{2}}{ }_{k+\frac{1}{2}}^{n}}{\Delta t}=0 \quad(I-B)$

$i^{n+\frac{1}{2}}-i^{n+\frac{1}{2}}$

$\frac{k_{k+\frac{1}{2}}-i{ }_{k-\frac{1}{2}}}{\Delta x}+\dot{G} \frac{v_{k}^{n+1}+v_{k}^{n}}{2}+\dot{C} \frac{v_{k}^{n+1}-v_{k}^{n}}{\Delta t}=0 \quad(I I-B)$

Formula (I-B) gives us:

$i_{k+\frac{1}{2}}^{n+\frac{1}{2}}=\left[\frac{L}{\Delta t}+\frac{\dot{R}}{2}\right]^{-1}\left\{\left[\frac{\dot{L}}{\Delta t}-\frac{\dot{R}}{2}\right] i_{k+\frac{1}{2}}^{n-\frac{1}{2}}-\frac{v_{k+1}^{n}-v_{k}^{n}}{\Delta x}\right\}$

$i_{k+\frac{1}{2}}^{n+\frac{1}{2}}=\sigma 1 *\left(v_{k}^{n}-v_{k}^{n+1}\right)+\theta 1 * i_{k+\frac{1}{2}}^{n-\frac{1}{2}}$

With:

$$
\begin{array}{rl}
\sigma 1=\left[\frac{L}{\Delta t}+\frac{\hat{R}}{2}\right]^{-1} & * \frac{1}{\Delta x} \text { and } \theta 1 \\
& =\left[\frac{\hat{L}}{\Delta t}+\frac{\hat{R}}{2}\right]^{-1} *\left[\frac{L}{\Delta t}-\frac{\hat{R}}{2}\right]^{1}
\end{array}
$$

We obtain the system:

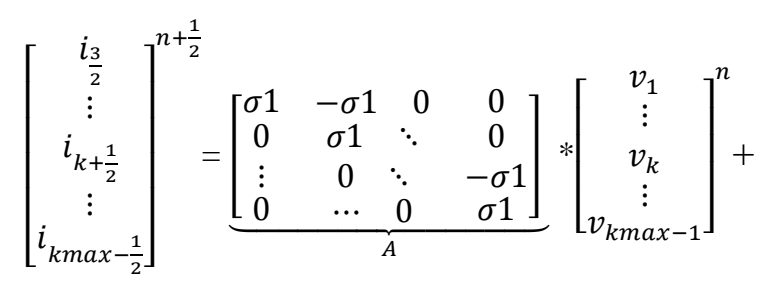

$$
\underbrace{\left[\begin{array}{cccc}
\theta 1 & 0 & 0 & 0 \\
0 & \theta 1 & \ddots & 0 \\
\vdots & 0 & \ddots & 0 \\
0 & \cdots & 0 & \theta 1
\end{array}\right]}_{\Theta 1} *\left[\begin{array}{c}
i_{\frac{3}{2}} \\
\vdots \\
i_{k+\frac{1}{2}} \\
\vdots \\
i_{k \max -\frac{1}{2}}
\end{array}\right]^{n-\frac{1}{2}}
$$

We obtain the matrix form:

$I^{n+\frac{1}{2}}=A * V^{n}+\Theta 1 * I^{n-\frac{1}{2}}$

Similarly, formula (II-B) gives us:

$v_{k}^{n+1}=\left[\frac{\dot{C}}{\Delta t}+\frac{\dot{G}}{2}\right]^{-1}\left\{\frac{i_{k-\frac{1}{2}}^{n+\frac{1}{2}}-i_{k+\frac{1}{2}}^{n+\frac{1}{2}}}{\Delta x}+\left[\frac{\dot{C}}{\Delta t}-\frac{\dot{G}}{2}\right] v_{k}^{n}\right\}$

$v_{k}^{n+1}=\sigma 2 *\left(i_{k-\frac{1}{2}}^{n+\frac{1}{2}}-i_{k+\frac{1}{2}}^{n+\frac{1}{2}}\right)+\theta 2 * v_{k}^{n}$

$\sigma 2=\left[\frac{\dot{C}}{\Delta t}+\frac{\dot{G}}{2}\right]^{-1} * \frac{1}{\Delta x}$ and $\theta 2=\left[\frac{\dot{C}}{\Delta t}+\frac{\dot{G}}{2}\right]^{-1} *\left[\frac{\dot{C}}{\Delta t}-\frac{\dot{G}}{2}\right]^{1}$

We obtain the system:

$\left[\begin{array}{c}v_{2} \\ \vdots \\ v_{k} \\ \vdots \\ v_{k \max -1}\end{array}\right]^{n+1}=\underbrace{\left[\begin{array}{cccc}\sigma 2 & -\sigma 2 & 0 & 0 \\ 0 & \sigma 2 & \ddots & 0 \\ \vdots & 0 & \ddots & -\sigma 2 \\ 0 & \cdots & 0 & \sigma 2\end{array}\right]}_{\mathrm{B}} *\left[\begin{array}{c}i_{\frac{3}{2}} \\ \vdots \\ i_{k+\frac{1}{2}} \\ \vdots \\ i_{k \max -\frac{1}{2}}\end{array}\right]^{n+\frac{1}{2}}+$

$\underbrace{\left[\begin{array}{cccc}\theta 2 & 0 & 0 & 0 \\ 0 & \theta 2 & \ddots & 0 \\ \vdots & 0 & \ddots & 0 \\ 0 & \cdots & 0 & \theta 2\end{array}\right]}_{\Theta 2} *\left[\begin{array}{c}v_{2} \\ \vdots \\ v_{k} \\ \vdots \\ v_{k \max -1}\end{array}\right]^{n}$

$V^{n+1}=B * I^{n+\frac{1}{2}}+\Theta 2 * V^{n}$

The conditions at both ends of the line are:

At $\mathrm{x}=0$ at the moment $\left(n+\frac{1}{2}\right) \Delta t$ we have:

$v_{1}^{n+1}=E_{1}^{n+1}-R_{A} i_{\frac{3}{2}}^{n+\frac{1}{2}}$

Or in case of current source:

$v_{1}^{n+1}=R_{A}\left(I s_{1}^{n+1}-i_{\frac{3}{2}}^{n+\frac{1}{2}}\right)$

Similarly, at $\mathrm{x}=\mathrm{L}: \quad v_{k_{\max }}^{n+1}=+R_{B} i_{k_{\max }-\frac{1}{2}}^{n+\frac{1}{2}}$

The validity of the method depends on the accuracy of calculations and the stability of the algorithm. The method is stable if we respect the following condition:

$\Delta t<\frac{\Delta x}{v_{p}}$ 
With $v_{p}$ is the speed of propagation on the studied transmission line.

B. Application of the FDTD method to the equations of a non-uniform transmission line

The equations of the nonuniform line are calculated from the generalized Ohm's law on an elementary section.

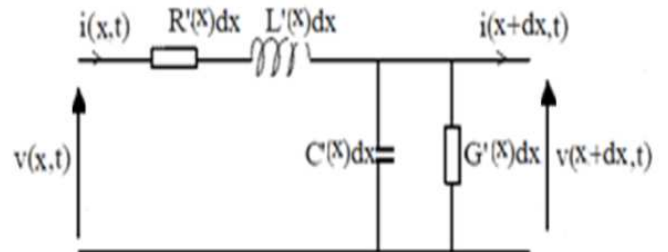

Fig. 4. Elementary section of a nonuniform transmission line

The parameters (R, L, C and G) in the case of a uniform line are constant along the line. In the case of a nonuniform transmission line, these elements are dependent on the geometry of the line and therefore are functions of $\mathrm{x}$.

Equations (I) and (II) become:

$$
\begin{aligned}
& \frac{\partial v(x, t)}{\partial x}+R^{\prime}(x) i(x, t)+L^{\prime}(x) \frac{\partial i(x, t)}{\partial t}=0 \\
& \frac{\partial i(x, t)}{\partial x}+G^{\prime}(x) v(x, t)+C^{\prime}(x) \frac{\partial v(x, t)}{\partial t}=0 \\
& i_{k+\frac{1}{2}}^{n+\frac{1}{2}}=\left[\frac{L^{\prime}{ }_{k+\frac{1}{2}}}{\Delta t}+\frac{R^{\prime}{ }_{k+\frac{1}{2}}}{2}\right]^{-1}\left\{\left[\frac{L^{L^{\prime}+\frac{1}{2}}}{\Delta t}-\frac{R^{\prime}{ }_{k+\frac{1}{2}}}{2}\right] i_{k+\frac{1}{2}}^{n-\frac{1}{2}}-\frac{v_{k+1}^{n}-v_{k}^{n}}{\Delta x}\right\} \\
& v_{k}^{n+1}=\left[\frac{C^{\prime}{ }_{k}}{\Delta t}+\frac{G^{\prime}{ }_{k}}{2}\right]^{-1}\left\{\frac{i_{k-\frac{1}{2}}^{n+\frac{1}{2}}-i_{k+\frac{1}{2}}^{n+\frac{1}{2}}}{\Delta x}+\left[\frac{C^{\prime}{ }_{k}}{\Delta t}-\frac{G^{\prime}{ }_{k}}{2}\right] v_{k}^{n}\right\}
\end{aligned}
$$

The formulation is similar to that of a uniform line with:

$$
\begin{aligned}
& \sigma 1 \mathrm{k}=\left[\frac{L_{k+\frac{1}{2}}^{\prime}}{\Delta t}+\frac{R_{k+\frac{1}{2}}^{\prime}}{2}\right]^{-1} * \frac{1}{\Delta x} \text { and } \\
& \theta 1 \mathrm{k}=\left[\frac{L_{k+\frac{1}{2}}^{\prime}}{\Delta t}+\frac{R_{k+\frac{1}{2}}^{\prime}}{2}\right]^{-1} *\left[\frac{L_{k+\frac{1}{2}}^{\prime}}{\Delta t}-\frac{R_{k+\frac{1}{2}}^{\prime}}{2}\right]^{1} \\
& \sigma 2 \mathrm{k}=\left[\frac{C^{\prime}{ }_{k}}{\Delta t}+\frac{G^{\prime}{ }_{k}}{2}\right]^{-1} * \frac{1}{\Delta x} \text { and } \\
& \theta 2 \mathrm{k}=\left[\frac{C_{k}^{\prime}}{\Delta t}+\frac{G_{k}^{\prime}}{2}\right]^{-1} *\left[\frac{C^{\prime}{ }_{k}}{\Delta t}-\frac{G^{\prime}{ }_{k}}{2}\right]^{1}
\end{aligned}
$$

Terms $\sigma 1 \mathrm{k}, \theta 1 \mathrm{k}, \sigma 2 \mathrm{k}, \theta 2 \mathrm{k}$ are functions of spatial position on the line.
C. Application of the FDTD method to equations of a multiwire non-uniform transmission line

We consider a two conductors line above a reference plane.

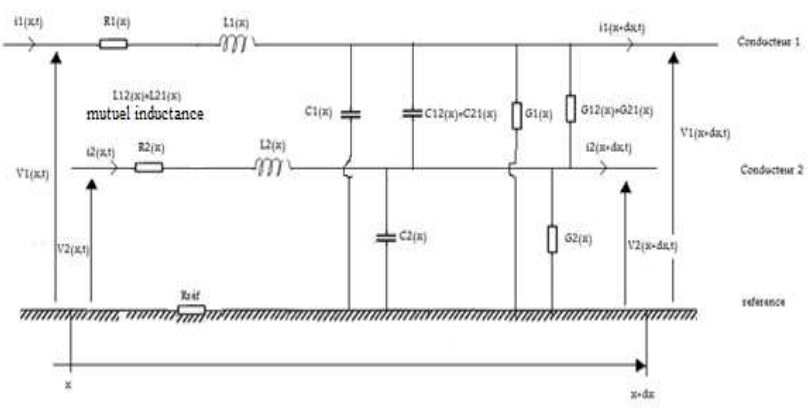

Fig. 5. Nonuniform line with two conductors

In this case the telegrapher equations become:

$$
\left\{\begin{array}{l}
\frac{\partial}{\partial x}\left(\begin{array}{l}
v 1(x, t) \\
v 2(x, t)
\end{array}\right)+R^{\prime}(x)\left(\begin{array}{l}
i 1(x, t) \\
i 2(x, t)
\end{array}\right)+L^{\prime}(x) \frac{\partial}{\partial t}\left(\begin{array}{l}
i 1(x, t) \\
i 2(x, t)
\end{array}\right)=0 \\
\frac{\partial}{\partial x}\left(\begin{array}{l}
i 1(x, t) \\
i 2(x, t)
\end{array}\right)+G^{\prime}(x)\left(\begin{array}{l}
v 1(x, t) \\
v 2(x, t)
\end{array}\right)+C^{\prime}(x) \frac{\partial}{\partial t}\left(\begin{array}{l}
v 1(x, t) \\
v 2(x, t)
\end{array}\right)=0
\end{array}\right.
$$

With $R^{\prime}(x), L^{\prime}(x), G^{\prime}(x)$ and $C^{\prime}(x)$ are matrices (2,

2) per unit length parameters:

$$
\begin{aligned}
& \mathrm{R}^{\prime}(\mathrm{x})=\left[\begin{array}{cc}
R^{\prime} 1+R^{\prime} r e f & R^{\prime} \text { ref } \\
R^{\prime} r e f & R^{\prime} 2+R^{\prime} r e f
\end{array}\right](x) ; \\
& \mathrm{L}^{\prime}(\mathrm{x})=\left[\begin{array}{cc}
L^{\prime} 1 & L^{\prime} 12 \\
L^{\prime} 21 & L^{\prime} 2
\end{array}\right](x) ; \\
& \mathrm{G}^{\prime}(\mathrm{x})=\left[\begin{array}{cc}
G^{\prime} 1+G^{\prime} 12 & -G^{\prime} 12 \\
-G^{\prime} 21 & G^{\prime} 1+G^{\prime} 21
\end{array}\right](x) ; \\
& \mathrm{C}^{\prime}(\mathrm{x})=\left[\begin{array}{cc}
C^{\prime} 1+C^{\prime} 12 & -C^{\prime} 12 \\
-C^{\prime} 21 & C^{\prime} 1+C^{\prime} 12
\end{array}\right](x)
\end{aligned}
$$

We set:

$\mathrm{V}(\mathrm{x}, \mathrm{t})=\left(\begin{array}{l}v 1(x, t) \\ v 2(x, t)\end{array}\right)$ and $\mathrm{i}(\mathrm{x}, \mathrm{t})=\left(\begin{array}{l}i 1(x, t) \\ i 2(x, t)\end{array}\right)$

We also obtain a formulation similar to that of a line formed of a single wire. And then terms $\sigma 1 \mathrm{k}$, $\theta 1 \mathrm{k}, \sigma 2 \mathrm{k}, \theta 2 \mathrm{k}$ will be matrices $(2,2)$.

In the case of a multiwire line with $\mathrm{n}$ conductors we apply the same formalism. The terms $\mathrm{R}^{\prime}(\mathrm{x})$, $\mathrm{L}^{\prime}(\mathrm{x}), \mathrm{G}^{\prime}(\mathrm{x}), \mathrm{C}^{\prime}(\mathrm{x}), \sigma 1 \mathrm{k}, \theta 1 \mathrm{k}, \sigma 2 \mathrm{k}, \theta 2 \mathrm{k}$ will be matrices (n, n).

\section{Application in the case of the propagation of lightning on an electric cable}


When a lightning strike a wire of a power electrical network, it behaves like a current injected into the conductor. This current is divided by half of both sides of the impact point, and each of these halves will propagate along the conductor. A direct lightning strike to a conductor, give very high intensity currents, the voltage waveform associated is characterized by large amplitudes of several MV order.

As we have said, the PLT allows the transmission of high rates information along the electric line, this type of cables varies between different networks. Figure (6) shows an example of used electric cables, 3 conductors (no1, 2 and 3) separated by inter-conductor distances (D12, D13 et D23) and a rotation angle $\varnothing$ that varies as a function of the length. Multiple successive sections of the cable allow the determination of a model that takes into account geometric variations.
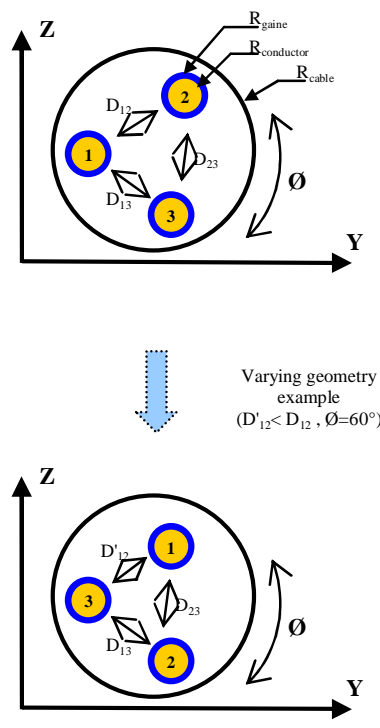

Fig. 6. Example showing a section of a three conductor wire

In this paper, we consider an energy cable composed of three twisted conductors (phase, neutral and earth). The different geometric parameters of the cable are:

$\mathrm{R}_{\text {conductor }}=5 \mathrm{~mm} ; \mathrm{R}_{\text {sheath }}=5.75 \mathrm{~mm} ; \mathrm{R}_{\text {cable }}=49$ $\mathrm{mm}$.

The cable is situated at 8 meters above the ground and has a total length of 1000 meters.
The current wave of lightning impact the first wire at $x=0$. The figure (7) shows the evolution of $\quad v(x, t)$ and $i(x, t)$ along conductors 1 and 3.

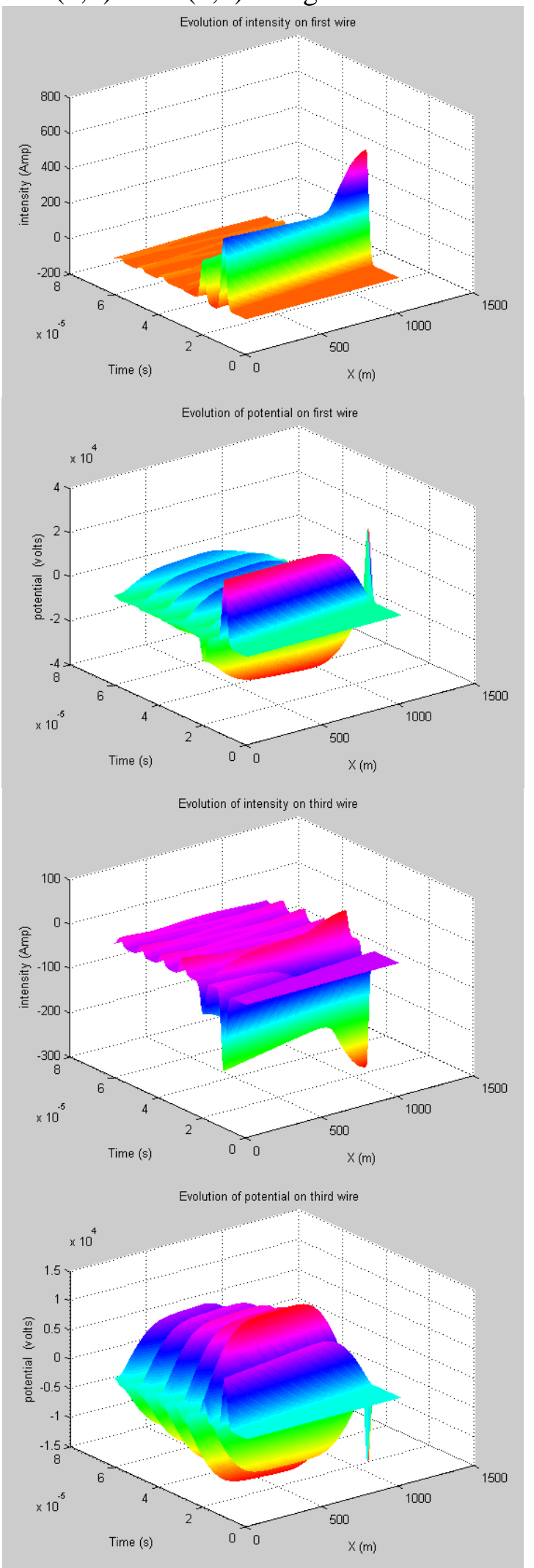




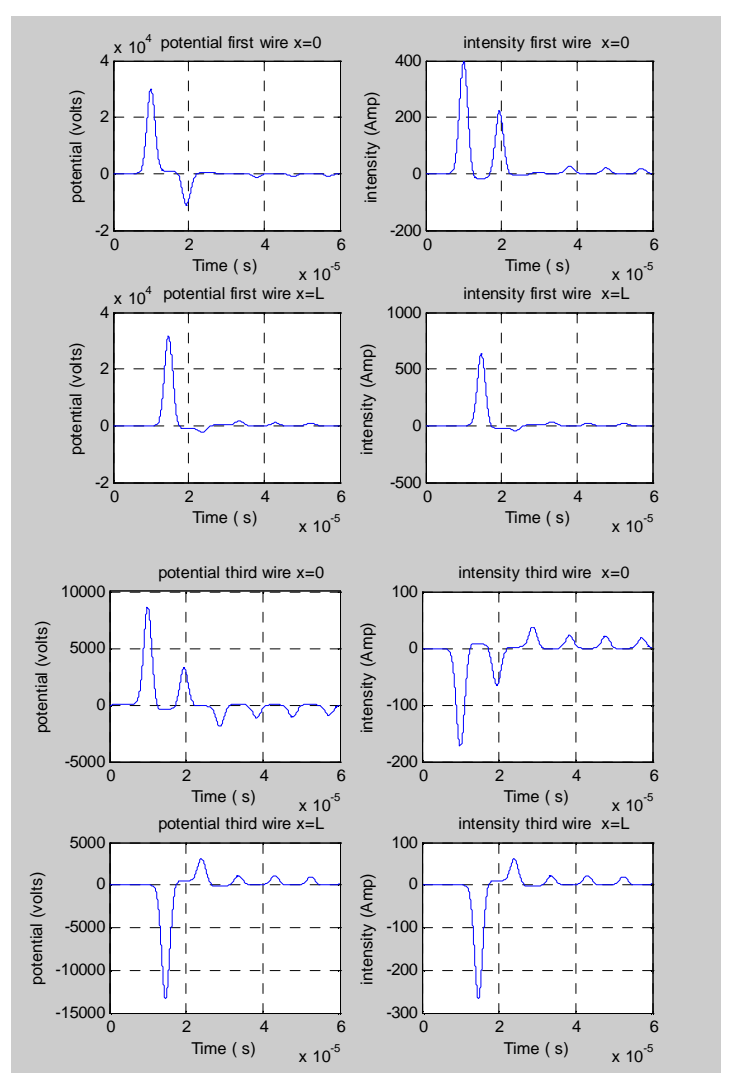

Fig. 7. Example showing evolution of $v(x, t)$ and $i(x, t)$

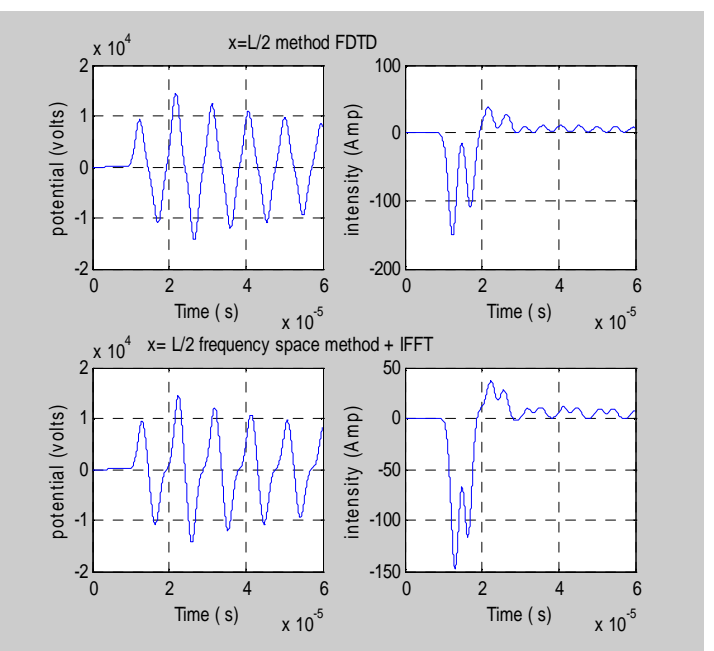

Fig. 8. v $(x, t)$ and i $(x, t)$ by FDTD \&Frequency method

\section{Conclusion}

We have proposed in this paper a method to simulate the propagation of an electrical wave along a multiwire line. The method is based on a model derived from the modified theory of lines. The electrical parameters are calculated at each position of the line according to the geometric data. The simulation method is performed in the time domain. This method uses the FDTD principle and the simulation is performed using MATLAB code. We present a comparison of results obtained by this method with those obtained from a simulation in the frequency domain followed by an IFFT. This second method has been validated by experiments in an antecedent study [2], [3]. The good adequacy of the results proves the validity of the method proposed in this paper.

\section{References}

[1] W. Shi and J. Fang, "Evaluation of closed-form crosstalk models of coupled transmission lines," IEEE Trans. vol. 22, pp.174-181, May 1999.

[2] Ghazi Bousaleh, Rafic Hage chehade and Fahd Hassoun. «Analytical approach for the study of radiated emission of hospital cabling system conveying a transmission PLT. » Bioinformatics and Medical Engineering, BIME journal 2009.

[3] Bousaleh G., Hassoun F. and Ibrahim T. « Study of a signal propagation over a transmission line having a non uniform » 978-1-4244-3834-1/09/\$25.00 @ ACTEA 2009 IEEE.

[4] Ye Chunfei and Li Erping: Analytical expressions for perunit-length parameters of finite length transmission lines with discontinuities, IEEE Trans on EMC, vol.44 (3), pp. 478-481, 2002.

[5] C. R. Pall, Analysis of Multi conductor Transmission Lines, New York: Wiley, 1994.

[6] contribution au développement d'un isolateur coplanaire à résonance par la méthode FDTD », Thèse par Gwénaël Poitau, INSA Lyon, 2002.

[7] F. Hassoun, "Étude de l'émission électromagnétique générée par une transmission haut débit sur un réseau de type XDSL ou PLC", Thèse de l'université Blaise Pascal de Clermont Ferrand - Spécialité : Électronique et systèmes - Novembre 2006.

[8] Résolution numérique des équations aux dérivées partielles », Sébastien Charnoz \& Adrian Daerr, Université Paris 7 Denis Diderot. 